Rammohan Sriramdas*, Shreevar Rastogi and Rudra Pratap

\title{
Design Considerations for Optimal Absorption of Energy from a Vibration Source by an Array of Harvesters
}

DOI 10.1515/ehs-2015-0021

Abstract: Dynamic vibration absorption is a passive technique for suppressing unintended vibrations. Optimal absorption of energy from a vibration source entails the determination of absorber parameters such as resonant frequency and damping. In the present work, we propose a method to obtain these parameters for a generic case of large number of identical vibration absorbers placed on a vibration source. We discuss an iterative procedure to find the optimum absorber resonant frequency and damping by minimizing the total energy absorbed by the system. We also analyse the influence of damping of the entire set of absorbers on the total energy absorbed and the effect of the absorber mass on the bandwidth of absorption. The proposed method is verified by analysing the response of a set of cantilever absorber beams placed on a vibrating cantilever plate. The resonant frequencies of the system with different number of absorbers are verified experimentally. We identify, using our method, the absorber mass, resonant frequency and damping of the absorber at which significant amount of energy supplied to the system flows into absorbers. A potential application of this method in the context of energy harvesting is the design of harvesters for a given vibration source. We emphasize through our work that monitoring energies in the system and optimizing them is both rational and vital for designing multiple harvesters that absorb energy from a given vibration source optimally.

Keywords: dynamic vibration absorber, energy absorption bandwidth, energy harvester, frequency ratio, mass ratio, vibration damping

\footnotetext{
*Corresponding author: Rammohan Sriramdas, Department of Mechanical Engineering, and Centre for Nano Science and Engineering, Indian Institute of Science, Bangalore-560012, India, E-mail: rammohan@mecheng.iisc.ernet.in

Shreevar Rastogi, Rudra Pratap, Department of Mechanical Engineering, and Centre for Nano Science and Engineering, Indian Institute of Science, Bangalore-560012, India
}

\section{Introduction}

A dynamic vibration absorber absorbs unintended vibrations passively, thereby attenuating the motion of a primary structure. The primary vibrating structure, whose vibrations are sought to be suppressed, together with the vibration absorber constitutes a system. The primary vibrating structure can be considered to be either damped or undamped. In both cases there exists an optimum absorber damping at which the energy transfer is optimum (Den Hartog 1985). Additionally, the optimal energy transfer is influenced by the absorber resonant frequency and the absorber mass. These two parameters are obtained from primary structure resonant frequency and mass by defining two performance parameters, namely, frequency ratio and mass ratio. The frequency ratio is defined as a ratio of the absorber resonant frequency to the primary structure resonant frequency. Similar definition is applicable to mass ratio.

The performance parameters of a system with undamped primary structure and a damped absorber are determined in closed form by Den Hartog (1985). A system with non-negligible damping in the primary structure is usually not amenable to analytical solutions for optimum parameters (Braun 2002). Hence, numerical solutions are used for optimizing the primary structure response. In practice, most primary vibrating structures are damped. It is observed that the damping characteristics of the primary structure significantly influence the performance parameters, and hence, the attenuation. Several researchers have addressed the determination of optimum parameters for systems with light damping in primary structure (Ghosh and Basu 2007; Wong and Cheung 2008; Liu and Coppola 2010). The typical procedure adopted in obtaining the parameters for such systems is the optimization of response of the primary structure as discussed by Warburton (1982). The frequency locus method that involves constructing graphical criteria for optimization is another method to determine the optimum parameters for a generic system (Thompson 1981 and Randall, Halsted, and Taylor 1981). Different configurations of absorbers are also studied for effective transmission of energy, e.g., see Bapat and 
Kumaraswamy (1979) and Najafi, Ashory, and Jamshidi (2009). A damping element placed directly between the absorber and the base is efficient compared to that placed between the primary and the absorber mass as observed by Liu and Liu (2005) and Liu and Coppola (2010). The optimum frequency ratio for a light to moderately damped primary structure is given in closed form by Ghosh and Basu (2007) and procedure for obtaining absorber damping for continuous systems is given by Esmailzadeh and Jalili (1998) and Thompson (2007). However, for systems with moderate to large primary structure damping, the errors in estimating the optimum parameters using approximate relations become unacceptable. Hence, there is a need for a simple, robust and quick method to determine the optimum performance parameters for a generic system. In the present work, we propose a method to obtain the optimal values by minimizing the energy supplied to the system, unlike the existing method of minimizing the primary structure response. This method of estimating the parameters keeps track of the energy flow within the system, and hence, indicates the possible amount of energy absorption directly. Furthermore, we obtain the optimum parameters for a generic case that has multiple identical vibration absorbers placed on a single vibrating structure.

The vibration energy absorbing harvesters placed on a vibrating surface resemble passive dynamic vibration absorbers. The focus in energy harvesting community has been in treating the harvester simultaneously as an energy harvester and a vibration absorber, e.g., see Brennan, Melo, and Lopes (2013), Faruque Ali and Adhikari (2013) and Lee et al. (2014). The energy absorbed by the harvesters is a significant performance metric in vibration energy harvesting. The design of energy harvesting system, including the characteristics of vibration source, requires the determination of performance parameters for optimal energy transfer. We propose a procedure to obtain the optimum performance parameters for the configuration of several identical harvesters mounted on a given damped vibration source. This would further enable us to determine a combination of optimum number of harvesters, and the system parameters like mass ratio, absorber damping and the frequency ratio that minimize the total energy absorbed by the system.

The concept of dynamic vibration absorption is well established. However, we propose a novel procedure in the context of energy harvesting for estimating the optimum harvester parameters. Two conditions are developed by optimizing the energy absorbed by the system and they are iteratively satisfied to get the refined values of the optimal parameters. The optimal parameters not only ensure that the energy drawn by the system is least but also improve the bandwidth of absorption which is very valuable for energy harvesting. Several design methodologies are studied to increase the bandwidth of energy harvesters working on different transduction mechanisms, e.g., see Yang et al. (2014), and Arrieta et al. (2013). However, we can increase the bandwidth of harvesters irrespective of the transduction mechanism by adopting the proposed design methodology for energy harvesters.

\section{Modeling of Absorber Array}

Absorber array consists of several damped spring mass subsystems placed on a primary vibration source. Each of the $n$ dynamic vibration absorbers is modeled as a subsystem consisting of mass $m_{i}$, spring $k_{i}$, and damper $c_{i}(i=1,2, \ldots n)$ attached to the primary structure. The primary vibration source is modeled as a subsystem consisting of mass $m_{0}$, spring $k_{0}$, and damper $c_{O}$. The schematic of the multi degree of freedom system under study is shown in Figure 1. Although, in practice, the primary structure represents the vibration source for harvesters, the complete system is analysed for a base excitation with displacement $x_{g}$. The governing equations of motion for $n+1$ degrees of freedom system do not directly reveal the behaviour of the system. However, we show that the equations reduce to an incredibly simple and lucid form, when all the absorbers are made identical. The equations of motion for a generic damped system with identical absorbers are shown in Appendix 1. Henceforth, the system under study consists of $n$ identical absorbers mounted on a damped primary structure. The system consists of two important resonant frequencies on either side of the

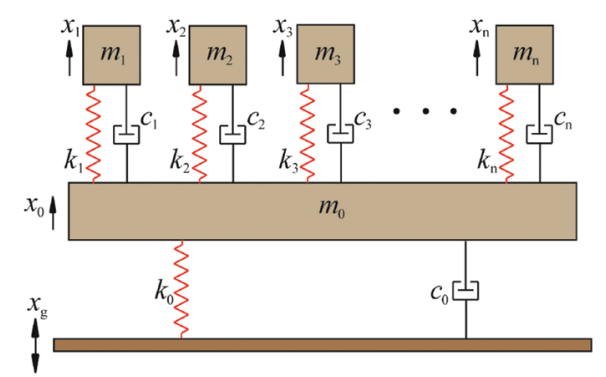

Figure 1: Schematic of vibration absorber array with $n$ identical absorbers mounted on a primary vibrating structure. The primary structure can be a huge vibrating plate with base excitation and absorbers represent several harvesters mounted on the structure for absorbing the energy. 
primary structure resonant frequency. At the first resonant frequency, all the absorber masses move in-phase with the primary mass, while at the second resonant frequency, they all move out-of-phase with the primary mass.

\section{Eigenvalues and Bandwidth of Absorption}

The two eigenvalues of the system as a function of primary structure resonant frequency $\omega_{0}$, and system parameters, namely, mass ratio defined as $m_{\mathrm{r}}=m_{\mathrm{i}} / m_{0}$, frequency ratio defined as $\alpha=\omega_{\mathrm{i}} / \omega_{0}$ and number of absorbers $n$, are given by

$$
\lambda_{1,2}=\omega_{0} \sqrt{\frac{1}{2}\left(\chi \mp \sqrt{\chi^{2}-4 \alpha^{2}}\right)},
$$

where $\chi=1+\alpha^{2} \beta, \beta=1+M_{r}$, and $M_{r}=n m_{r}$.

The effective mass ratio, $M_{\mathrm{r}}$, is defined as the product of mass ratio, $m_{\mathrm{r}}$ and the number of absorbers, $n$. It can be observed from eq. [1] that the eigenvalues, normalized with respect to the resonant frequency $\omega_{0}$ of the primary structure, are functions of frequency ratio, $\alpha$, and the effective mass ratio, $M_{\mathrm{r}}$. The normalized eigenvalues as a function of plausible effective mass ratios up to 0.5 and typical frequency ratios between 0.5 and 1.5 are shown in Figure 2. The first and second normalized resonant frequencies are shown in solid and dotted lines respectively. It can be observed from Figure 2 that the first resonant frequency is very sensitive to frequency ratio when $\alpha<1$, while the second resonant frequency is sensitive to frequency ratio when $\alpha>1$ for all mass ratios. The resonant frequencies, in case of a two degree of freedom system $(n=1)$, can be directly determined from the plot for a

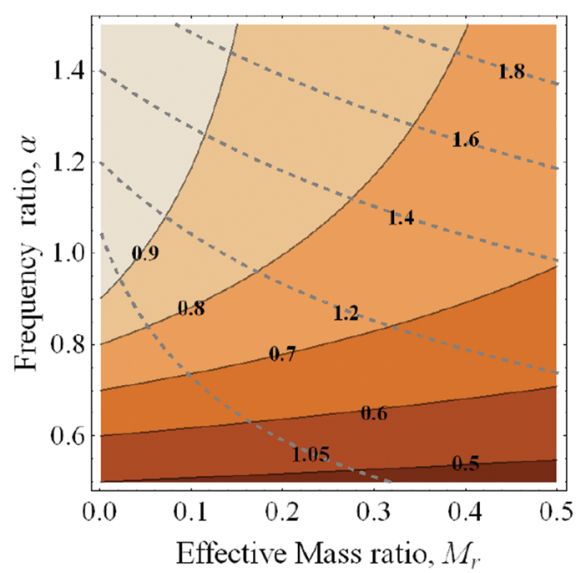

Figure 2: Eigenvalues of the system normalized with respect to the primary structure resonant frequency $\omega_{0}$. The figure shows that both frequencies increase as frequency ratio and effective mass ratio increase. given set of frequency ratio and mass ratio. However, for a system consisting of $n$ identical absorbers, the two eigen frequencies are determined appropriately using the effective mass ratio $M_{\mathrm{r}}$. Let the normalized eigenvalues be defined as $\gamma_{1,2}=\lambda_{1,2} / \omega_{0}$. From the eigenvectors, the displacement of the secondary absorber subsystem corresponding to the two eigenvalues is given as

$$
x_{i 1,2}=x_{0} \alpha^{2} /\left(\alpha^{2}-\gamma_{1,2}^{2}\right) \text {. }
$$

It can be observed from eq. [2] that the absorber displacement $x_{\mathrm{i}}$, normalized with respect to the primary structure displacement $x_{0}$ for both modes increases with decreasing mass ratio.

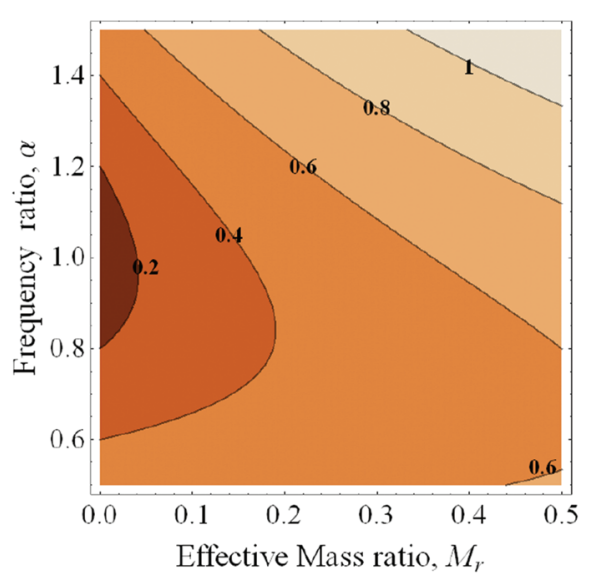

Figure 3: The difference between the two frequencies of the system normalized with the primary structure resonant frequency $\omega_{0}$. The difference increases with increasing frequency ratio and effective mass ratio.

The difference between the two normalized eigenvalues (Figure 3) can be a potential bandwidth of energy absorption if appropriate values of frequency ratio and absorber damping are selected. Although eigen analysis allows one to select a pair of $M_{\mathrm{r}}$ and $\alpha$ for bandwidth of absorption, it would be observed in the subsequent sections that optimum value of the frequency ratio $\alpha$ depends on the absorber damping. Hence, Figure 3 can be used to define a range for either $M_{\mathrm{r}}$ or $\alpha$ for the selected bandwidth of absorption.

\section{Energies in Vibration Absorber Array System}

The base excitation to the system is assumed to be a sinusoidal displacement of amplitude $u_{\mathrm{g}}$ at frequency $\omega$. The steady state displacements of the primary and the secondary absorber are assumed to be sinusoidal 
quantities of amplitudes $u_{0}$ and $u_{1}$ with the corresponding phase differences of $\theta_{0}$ and $\theta_{1}$ respectively. The displacements $x_{0}$ and $x_{1}$ derived from equations of motion are given in Appendix 1. The energies in the system are determined by integrating over one cycle the governing equations of motion multiplied by appropriate velocities. The only non-zero energies over one cycle are the dissipation energies in primary structure damper, secondary absorber dampers, and the input energy. The energy dissipated in primary damper $D E_{O}$, all the secondary dampers $D E_{n}$, and the input energy $I E$ are given respectively as

$$
\begin{gathered}
D E_{0}=\pi c_{0} u_{0}^{2} \omega, \\
D E_{n}=n \pi c_{1} \omega\left(u_{0}^{2}+u_{1}^{2}-2 u_{0} u_{1} \cos \left(\theta_{0}-\theta_{1}\right)\right) \quad \text { and } \\
I E=\pi m_{0} u_{g} \omega^{2}\left(u_{0} \sin \theta_{0}+m_{r} n u_{1} \sin \theta_{1}\right) .
\end{gathered}
$$

The objective is to determine optimal parameters that minimize the total energy absorbed by the system. The method starts by selecting an appropriate mass ratio depending on the bandwidth of energy absorption. Then determine the absorber damping and the frequency ratio by minimizing the system energies. The effect of nonoptimized parameters on energy input is illustrated through an example. Consider a primary vibrating system with mass, damping ratio and resonance as $1 \mathrm{~g}, 0.0001$ and $10 \mathrm{~Hz}$, respectively. In order to have a $2 \mathrm{~Hz}$ bandwidth of absorption, the upper limit on the effective mass ratio is less than 0.04 (Figure 3). Let the number of vibration absorbers, $n$, be 10 and mass ratio, $m_{r}$, be 0.003 . When the absorber resonant frequency is matched with that of the primary structure $(\alpha=1)$, the dependence of input energy on absorber damping, $\zeta_{1}$ and normalized drive frequency, $r_{O}$, defined as $r_{O}=\omega / \omega_{0}$ is shown in Figure 4.

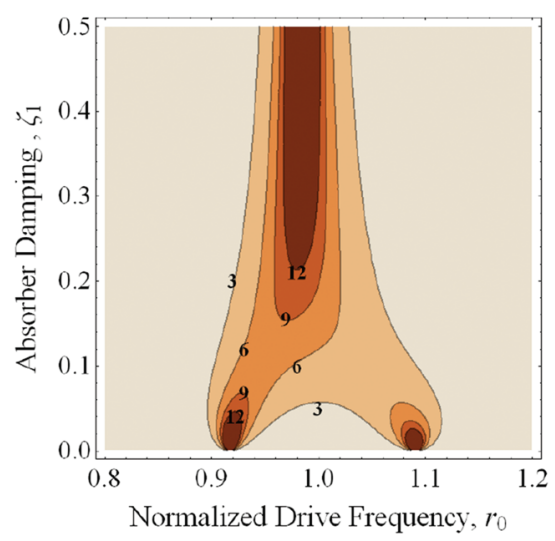

Figure 4: Dependence of the input energy on $r_{0}$ and $\zeta_{1}$ when swept at $1 \mathrm{~m} / \mathrm{s}^{2}$ for $\alpha=1$. The input energy absorbed by the system $(\mu \mathrm{J})$ is high at two frequencies below a certain value of absorber damping and at a single frequency above it.
It can be observed from Figure 4, which is plotted for $\alpha=1$ that considerable amount of energy is pumped into the system at selected frequencies of excitation and absorber damping $\zeta_{1}$. However, there exists a particular absorber damping $\zeta_{1}$ at which sizable amount of energy is pumped into the system over a larger range of frequencies. It can be observed from Figures 4 and 5 that there exist two significant performance parameters, namely, absorber damping $\zeta_{1}$ and frequency ratio $\alpha$ that influence the bandwidth of energy absorption. The procedure for selection of mass ratio and determination of the two parameters $\alpha$ and $\zeta_{1}$ is described in subsequent sections.

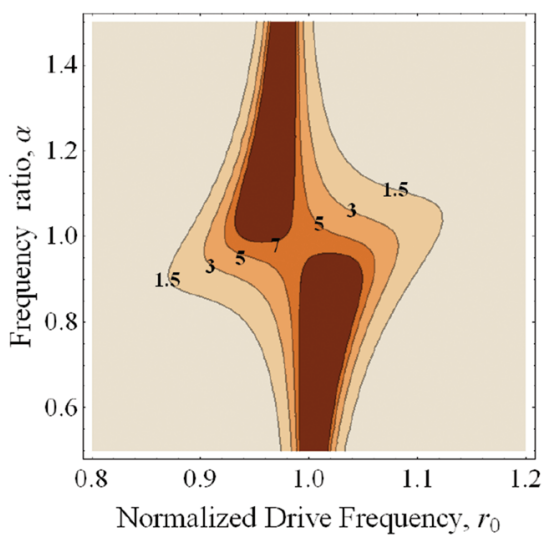

Figure 5: Dependence of input energy $(\mu \mathrm{J})$ on frequency ratio and normalized drive frequency at $\zeta_{1}=0.12$. The input energy $(\mu \mathrm{J})$ absorbed by the system depends on both absorber damping and frequency ratio.

\section{Selection of Mass Ratio}

It can be noticed from Figure 3 that difference between the two frequencies increases with increase in the effective mass ratio. As mentioned previously, the difference between the two frequencies represents the energy absorption bandwidth provided the frequency ratio and absorber damping are optimized. Moreover, bandwidth is limited by the effective mass ratio (Figure 3). Hence, the effective mass ratio for the system is selected by noting the amount of frequency difference sought between the two resonant modes. Alternatively, an upper limit on the effective mass ratio is governed by the required bandwidth of operation. Once the frequency difference is defined, an appropriate mass ratio from Figure 3 is selected and used in the subsequent energy optimization to obtain the optimum values of frequency ratio, $\alpha$ and absorber damping, $\zeta_{1}$. 


\section{Procedure for Determining Optimum $\zeta_{1}$ and $\alpha$}

At small values of $\zeta_{1}$, the energy absorbed by the system is high at two different values of frequencies (Figure 4). However, as $\zeta_{1}$ increases the two peaks merge reducing the bandwidth. If the system has undamped primary structure, there exist hinge points, called fixed points as described in Den Hartog (1985), and Braun (2002), that have constant energy irrespective of the absorber damping and allow the determination of $\alpha$ with relative ease. As the system under consideration has damped primary structure, an iterative procedure is adopted for estimating the two parameters. Firstly, the normalized drive frequencies corresponding to the optimum input energy are determined before estimating $\zeta_{1}$ and $\alpha$. The input energy is differentiated with respect to the normalized drive frequency, $r_{O}$ and is equated to zero to obtain an expression for determining the drive frequencies at which the energy has extrema.

$$
\begin{gathered}
\frac{\partial I E}{\partial r_{0}}=0 \\
3 p^{4}-p^{3}\left(2 \chi-4 \alpha^{2} \beta^{2} \zeta_{1}^{2}\right)-p^{2}\left(\chi^{2}+2 \alpha^{2}\left(1-4 \beta \zeta_{1}^{2}\right)\right) \\
+6 p \alpha^{2}\left(\chi-2 \zeta_{1}^{2}\right)-5 \alpha^{4}=0
\end{gathered}
$$

where $p=r_{0}^{2}$. For a given $\alpha$, eq. [6] results in only three real and positive roots for normalized drive frequency $r_{0}$. Let the three real positive roots be $r_{1}, r_{2}$ and $r_{3}$ such that $r_{1}<r_{2}<r_{3}$. It is observed from Figure 4 that the input energy is maximum at $r_{1}$ and $r_{3}$, while it is minimum at $r_{2}$ as $\zeta_{1}$ is increased from zero. Estimation of these three drive frequencies assumes that the primary structure damping $\zeta_{0}$ is negligible. Although one can determine the expression in eq. [6] by including the primary structure damping, the brevity of eq. [6] would be lost at the expense of marginal contribution to the accuracy of drive frequencies. However, subsequent optimization procedure includes the primary structure damping, $\zeta_{0}$, even if it is insignificant. The energy dissipated by both primary and secondary absorbers has maxima at $r_{1}$ and $r_{3}$. The first condition is obtained by equating the difference between the absorber energies evaluated at $r_{1}$ and $r_{3}$ to zero as

$$
\left.D E_{1}\right|_{r_{0}=r_{1}}-\left.D E_{1}\right|_{r_{0}=r_{3}}=0 .
$$

It may be noted that $r_{1}$ and $r_{3}$, obtained from eq. [6] are functions of unknowns $\zeta_{1}$ and $\alpha$. The absorber damping $\zeta_{1}$ also influences the energy in primary structure. Thus a second condition is derived by differentiating the energy absorbed by primary structure $D E_{O}$ with respect to $\zeta_{1}$ as

$$
\frac{\partial D E_{0}}{\partial \zeta_{1}}=0
$$

$$
\begin{aligned}
& r_{0}^{6} \zeta_{0}+r_{0}^{4}\left(2 \alpha \zeta_{1}\left(\beta-2(1+\beta) \zeta_{0}^{2}\right)-2 \alpha^{2} \beta \zeta_{0}\left(1+2 \beta \zeta_{1}^{2}\right)\right) \\
& +r_{0}^{2}\left(\alpha^{4} \beta^{2} \zeta_{0}+2 \alpha^{3} \beta \zeta_{1}\left(4 \zeta_{0}^{2}-\beta\right)-\alpha \zeta_{1}(1+\beta)\right)+2 \alpha^{3} \beta \zeta_{1}=0
\end{aligned}
$$

\section{Summary of Iteration Steps}

1. Select the required bandwidth of energy absorption for $n$ number of harvesters, and pick the appropriate mass ratio from eigenvalues (Figure 3).

2. Determine the real positive roots of eq. [6] for $r_{0}$ in terms of unknowns $\alpha$ and $\zeta_{1}$ in the order $r_{1}<r_{2}<r_{3}$.

3. Substitute either $r_{1}$ or $r_{3}$ in second condition given in eq. [8] assuming $\alpha=1$ to determine the unknown $\zeta_{1}$.

4. Using $\zeta_{1}$ determined in step 3, calculate the unknown $\alpha$ from the first condition given in eq. [7].

5. Use the value of $\alpha$ calculated in step 4 and repeat step 3 with new $\alpha$ to refine $\zeta_{1}$.

6. Repeat steps 3 through 5 to arrive at the converged optimal values of $\alpha$, and $\zeta_{1}$.

\section{Illustration}

Consider the system described in the previous section that consists of ten absorbers with individual mass ratio of 0.003 and an anticipated bandwidth less than $2 \mathrm{~Hz}$. The optimum values of the absorber damping ratio and the frequency ratio are determined iteratively by following the previously outlined steps.

Table 1: Values of $r_{1}, r_{3}$, frequency ratio $\alpha$ and absorber damping $\zeta_{1}$ after every iteration.

\begin{tabular}{lrrrr}
\hline Iteration no. & $\alpha$ & $\boldsymbol{\zeta}_{\mathbf{1}}$ & $\boldsymbol{r}_{\mathbf{1}}$ & $\boldsymbol{r}_{\mathbf{3}}$ \\
\hline 0 & 1 & - & - & - \\
1 & 0.975880 & 0.094535 & 0.936296 & 1.042278 \\
2 & 0.976300 & 0.085053 & 0.929415 & 1.050444 \\
3 & 0.976295 & 0.085197 & 0.929507 & 1.050333 \\
4 & 0.976294 & 0.085194 & 0.929506 & 1.050334 \\
5 & 0.976294 & 0.085194 & 0.929506 & 1.050334 \\
\hline
\end{tabular}

It can be observed from Table 1 that the values converge within a very few iterations. The performance parameters thus determined are used to design the system. The energy absorbed by all the absorbers corresponding to the optimum frequency ratio $(\alpha=0.976)$ as shown in last iteration of Table 1 is plotted in Figure 6 for increasing values of absorber damping. It can be observed from the figure that the energy absorbed is made equal at both the resonant frequencies as per the first condition. Moreover, 


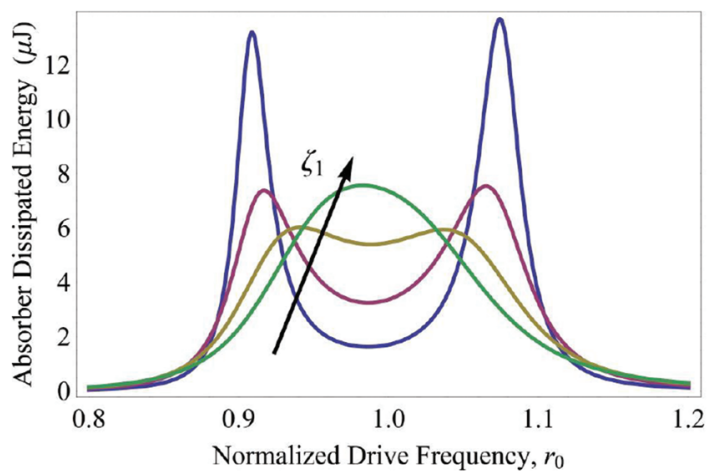

Figure 6: The figure shows the energy absorbed by absorbers operated at the optimal frequency ratio $\alpha=0.976$ for different values of absorber damping $-0.03,0.06,0.1$ and 0.14 .

the energy close to the optimum $\zeta_{1}$ has a wider bandwidth and equal values of energies at both the frequencies.

The influence of the frequency ratio $\alpha$ on the energy absorbed by all the absorbers that are designed to have optimum absorber damping $\left(\zeta_{1}=0.085\right)$ is shown in Figure 7. It is thus essential to select both frequency ratio and absorber damping appropriately, for efficient absorption of energy over a predefined bandwidth which is crucial in energy harvesters. Typically, several harvesters are mounted on a given vibrating surface in order to effectively absorb the energy. Designing an array of identical absorbers is an alternative for implementing the optimized absorber damping, if a single damper is infeasible. For a given number of harvesters, the damping (sum of electrical and mechanical damping) of the harvester can be tuned to achieve wider bandwidth, and optimal energy of absorption.

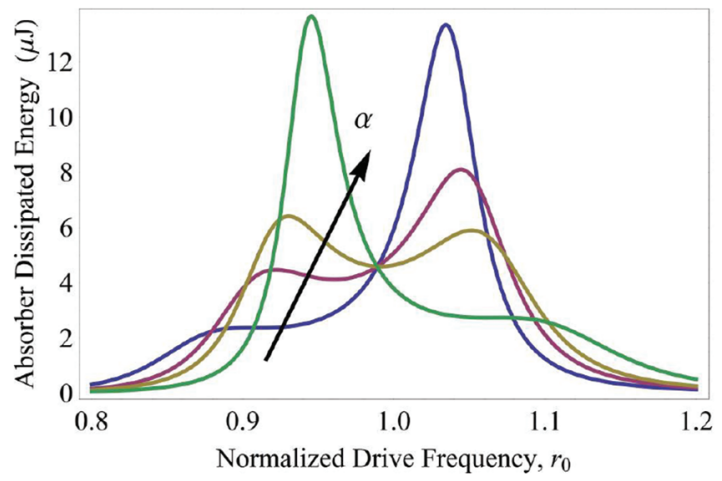

Figure 7: The figure shows the energy absorbed by absorbers designed to have optimal damping of 0.085 for different values of frequency ratio $-0.9,0.95,0.98$, and 1.05 .

The bandwidth of absorption as a function of the absorber damping evaluated at the optimum frequency ratio is shown in Figure 8. When operated at the optimum

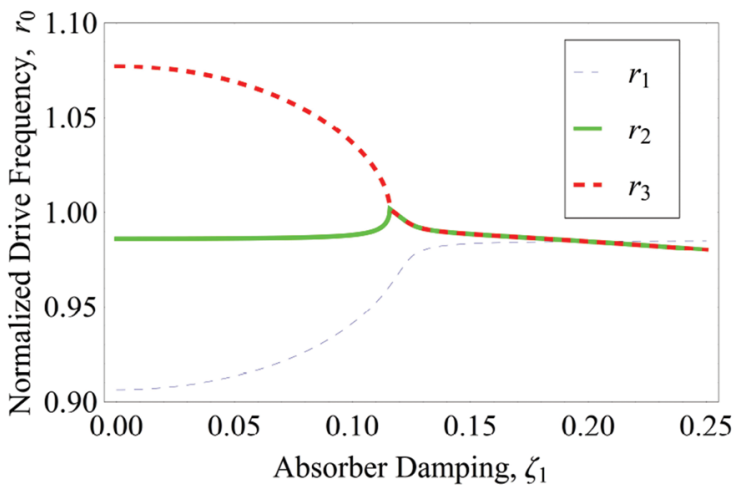

Figure 8: The figure shows the bandwidth of energy absorption as a function of absorber damping. These are determined from eq. [6] for the optimal value of $\alpha$. Bandwidth reduces beyond the optimal value of $\zeta_{1}$.

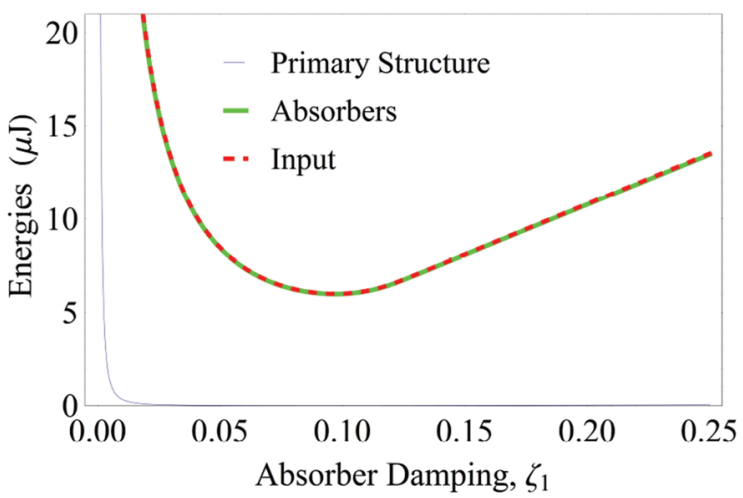

Figure 9: The three energies of the system are shown as a function of $\zeta_{1}$ at optimized $\alpha$. Absorbers take away significant amount of input energy at any absorber damping; however, all the energies are minimum at the optimum value of $\zeta_{1}$.

frequency ratio $\alpha$, much of the energy supplied to the system is absorbed by the harvesters as shown in Figure 9, which further indicates that the energy absorbed by primary structure is made negligible. However, energy supplied to the system is minimized at a particular damping that corresponds to the optimum absorber damping. The optimum absorber damping ensures that the energy drawn by the system from ambience is minimum provided the absorber or the harvesting system is designed to have the optimum frequency ratio.

\section{Experimental Corroboration}

The feasibility of attaining the optimum performance parameters is studied by performing controlled experiments on 
a primary vibrating structure. A steel plate of $300 \mu \mathrm{m}$ thickness, clamped along an edge is considered as the primary structure. The length and width of the structure are $51 \mathrm{~mm}$ and $40 \mathrm{~mm}$ respectively. Rayleigh Ritz method is employed to determine the plate resonances. The shape function corresponding to the fundamental mode is obtained by generating boundary characteristic orthogonal polynomials as described in Chakraverty (2009). Using the first 15 orthogonal polynomial functions, the first resonant frequency is determined precisely to be $90.3 \mathrm{~Hz}$ and verified experimentally to be $89.9 \mathrm{~Hz}$. The structure is subjected to sinusoidal base excitation using an electromagnetic shaker (LDS, V406/8-PA100E, Royston, UK) around the resonance to obtain primary structure damping $\zeta_{0}$. The damping ratio is determined from the response using half power bandwidth method to be 0.004 . The vibration absorbers are made in the form of cantilevers mounted on the edge opposite to the clamped side of the plate. In order to observe the effect of multiple absorbers, the system consisting of three such absorbers is tested. The absorber beams are made using steel and brass materials of different thicknesses to get different mass ratios. The vibrational response of the absorbers is measured using a laser doppler vibrometer (PDV-100, Polytec GmbH, Germany). The absorber system consisting of three identical absorber beams mounted on a primary structure is shown in Figure 10.

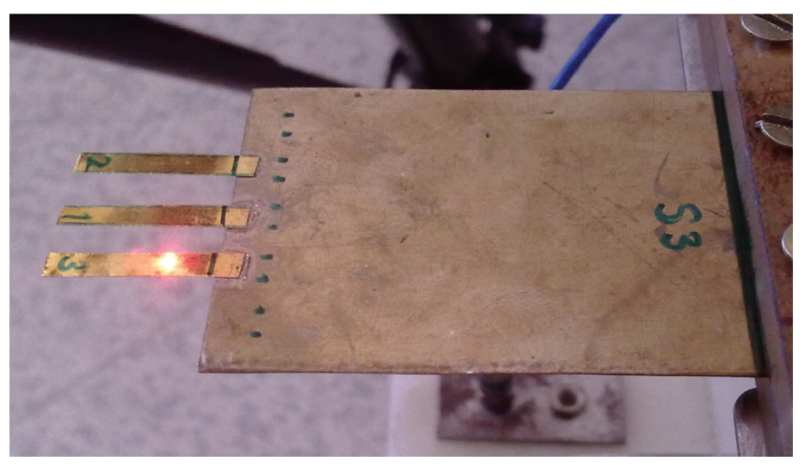

Figure 10: The figure shows three beams of brass forming three identical absorbers mounted on a cantilever steel plate that in turn is mounted on a shaker.

\section{Determination of Mass Ratio}

Estimating the mass ratio for continuous systems is not as straight forward as finding the frequency ratio. The frequency ratio can directly be determined from material properties and dimensions of the structures. However, mass ratio for continuous systems is obtained by matching the eigenvectors described by eq. [2]. Let the length of the primary structure be $l$ and the total length up to absorber tip be $\mathrm{L}$. In order to determine the mass ratio approximately, consider shape functions in the two segments $(i=1,2)$ in the form of polynomials $\psi_{i}=\sum_{j=2}^{4} C_{i j} x^{j}$. The coefficients are determined by employing the boundary conditions at the interface and conditions at the tip as governed by eigenvectors defined in eq. [2]. For the assumed shape functions $\psi_{\mathrm{i}}$, mass ratio is numerically determined using the expression

$$
\Psi\left(m_{r}\right) \equiv \bar{m}_{1} m_{r} \int_{0}^{l} \psi_{1}^{2}\left(m_{r}\right) d x-\bar{m}_{2} \int_{l}^{L} \psi_{2}^{2}\left(m_{r}\right) d x=0,
$$

where $\bar{m}_{1}$ and $\bar{m}_{2}$ are mass per unit length for both primary and secondary absorber subsystems respectively normalized with respect to unit mass in order to make eq. [9] dimensionless.

The value corresponding to zero of the nondimensional eq. [9] would give the required mass ratio. For the absorber system consisting of 3 steel absorbers each of length $28 \mathrm{~mm}$, width $2.5 \mathrm{~mm}$, thickness $100 \mu \mathrm{m}$ resulting in $\alpha$ of 1.02, the mass ratio is estimated to be 0.126 (Figure 11). Experimentally, mass ratio for the system is found to be 0.106 by matching the eigen frequency (Figure 2). The difference between the mass ratios evaluated numerically and estimated experimentally is about $18 \%$ and the accuracy improves by employing exact shape functions for continuous structures. Once the mass ratio is determined, the optimum values of frequency ratio and absorber damping for optimal energy transfer are obtained by following the procedure outlined in the previous section.

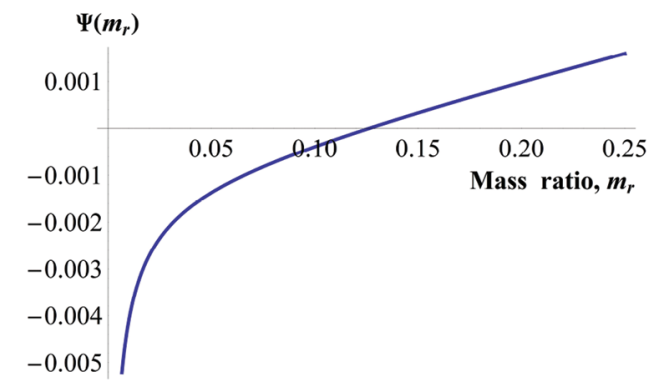

Figure 11: The plot shows the function $\psi\left(m_{r}\right)$ vs $m_{r}$. The zero of the function gives the value of mass ratio. The value corresponding to the approximate shape functions is 0.126 .

The behaviour of the system is studied by performing tests on the same primary structure with two different sets of absorbers. The objective is to determine the 
existing performance parameters in each case so as to select the most suitable absorber set for the given primary structure. The absorbers are designed appropriately and are fabricated to achieve the target configuration. In case of inadvertent deviations from optimal values owing to fabrication limitations and absorber damping mismatch, experimental observations are verified with the proposed theory. Each absorber set consists of three identical absorbers made of a particular material and specified thickness. Steel sheet of thickness $100 \mu \mathrm{m}$ and brass sheet of thickness $50 \mu \mathrm{m}$ are used to fabricate the absorber sets. The test results in each case are described as follows.

\section{Absorber Set 1}

The primary structure resonance is measured to be $89.9 \mathrm{~Hz}$ and the damping ratio obtained using half power bandwidth method is 0.004 . The primary structure is subjected to a sinusoidal sweep of $0.1 \mathrm{~g}_{n}$ acceleration from $85 \mathrm{~Hz}$ to $94 \mathrm{~Hz}$ at $0.3 \mathrm{Oct} / \mathrm{min}$. Three steel absorbers of length 28 $\mathrm{mm}$ each are mounted on the primary structure. The resonant frequency of each absorber is found to be $91.14 \mathrm{~Hz}$. Thus frequency ratio is 1.01 in the present configuration. When a single absorber is used, the two resonant frequencies are $78.97 \mathrm{~Hz}$ and $106.69 \mathrm{~Hz}$ resulting in the mass ratio of 0.087 (Figure 2). When absorbers are increased to 3, the two resonant frequencies move to $68.91 \mathrm{~Hz}$ and $113.8 \mathrm{~Hz}$ as shown in Figure 12. Consequently the mass ratio increases to 0.11 (Figure 2). As noticed from Figure 3, decrease in $\alpha$ would not help in decreasing the difference between the

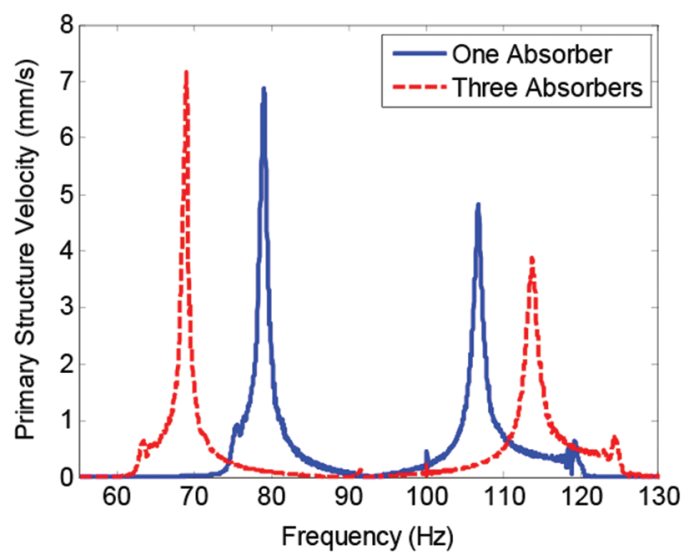

Figure 12: The figure shows the response of the primary structure for a single absorber and 3 absorbers. Separation between the frequencies increases with absorbers because of increasing mass ratio.

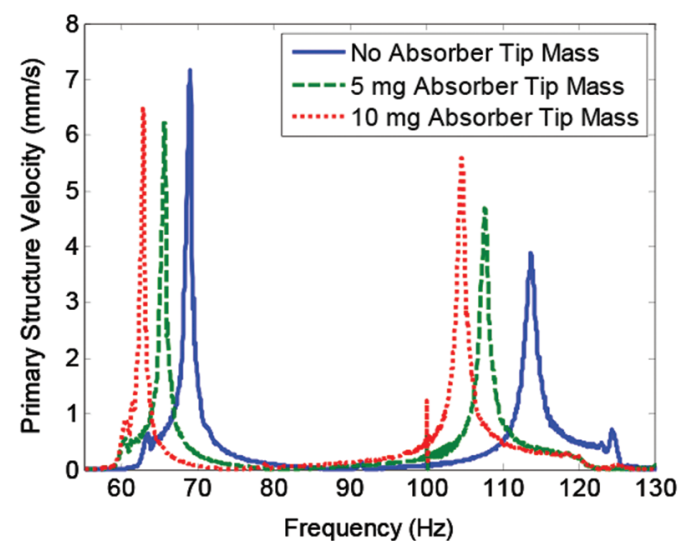

Figure 13: The figure shows the response of the primary structure with absorber mass of $10 \mathrm{mg}, 5 \mathrm{mg}$ and without absorber mass. The difference between the frequencies does not reduce significantly even though $\alpha$ is changing from 0.98 to 1.06 (Figure 3).

two frequencies. This is evident from Figure 13, where the response for two values of end masses is shown. The addition of tip mass to an absorber alters the frequency ratio significantly over the mass ratio. Hence, difference between the two frequencies does not decrease, rather remains almost constant with absorber tip mass.

\section{Absorber Set 2}

In order to arrive at a reasonable difference between the two frequencies, mass ratio of the system is decreased. As the equivalent mass of primary system cannot be altered, the absorber mass is reduced by using thinner beams. The absorbers are made out of $50 \mu \mathrm{m}$ brass sheet with length $16.6 \mathrm{~mm}$ giving a resonant frequency of 94 $\mathrm{Hz}$ that results in a frequency ratio of 1.05 . The corresponding mass ratio is estimated using eq. [9] to be 0.036 and is verified experimentally to be 0.030 by matching the resonant frequency in Figure 2. The response of the system for a sine sweep from $75 \mathrm{~Hz}$ to $110 \mathrm{~Hz}$ at $0.08 \mathrm{~g}_{\mathrm{n}}$ input is shown in Figure 14. The amplitudes at both peaks are not equal due to a higher value of $\alpha$ (Figure 7). The amplitude of the absorber measured at $2 / 3^{\text {rd }}$ distance from the absorber tip is compared with that of the primary structure in Figure 14. Clearly, the reduced mass ratio from the previous set of absorbers has brought the frequency difference down from $45 \mathrm{~Hz}$ to $16 \mathrm{~Hz}$. The damping ratio of the absorber is 0.0012 and the frequency ratio is 1.05 . The corresponding energies are calculated based on the experimental response and shown in Figure 15. 


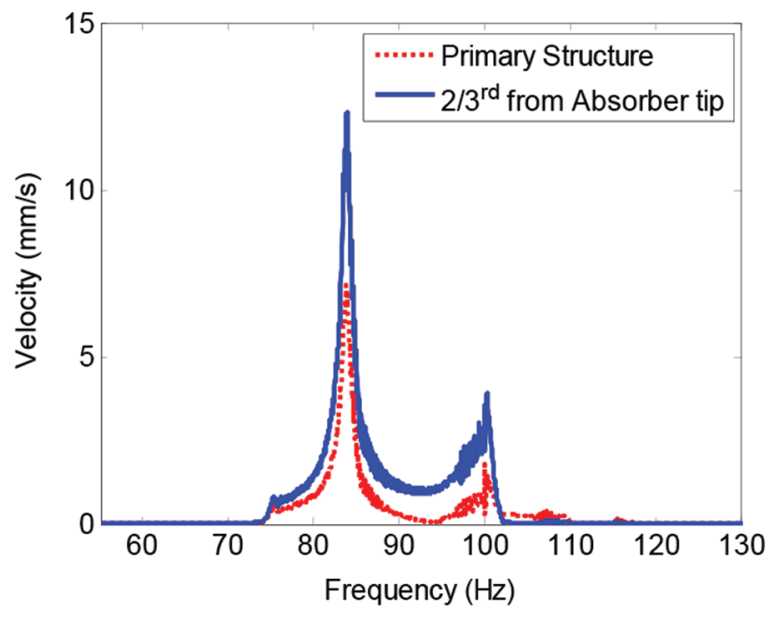

Figure 14: The figure shows response of the system. Primary structure response is measured at the free end while absorber response is measured at $2 / 3^{\text {rd }}$ distance from the absorber free end due to measurement limitations.

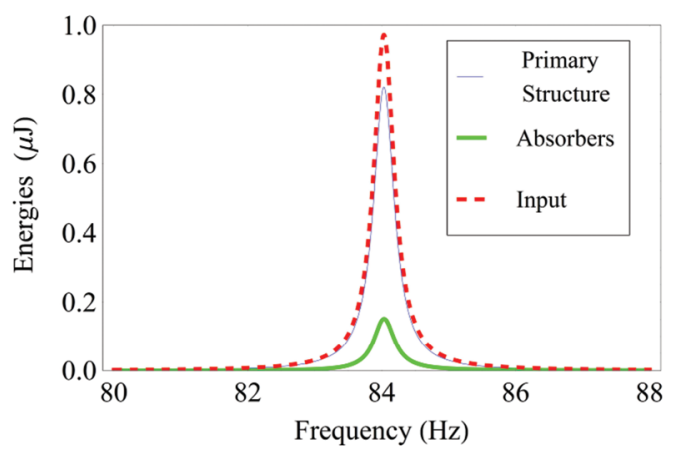

Figure 15: The figure shows energies in the system for the first mode. Much of the input energy is absorbed by the primary structure itself in case of the system with non-optimized parameters.

It is observed that the energy absorbed by primary structure is much higher than that of absorbers at either frequency. Although the amplitudes of absorbers are much larger than that of the primary structure, the energy absorbed by absorbers is much smaller when compared with the input energy. This situation is mitigated by operating the system at optimal values of frequency ratio and absorber damping. The optimal $\alpha$ and $\zeta_{1}$ for both sets of absorbers are obtained iteratively as shown in Table 2. The energy absorbed by the system operating at the optimum value of frequency ratio $\alpha$ as a function of absorber damping $\zeta_{1}$ is shown in Figure 16. It is essential to design absorbers or harvesters to have optimal performance parameters in order to decrease the burden on the vibration source.
Table 2: Optimal parameters extracted for absorber sets 1 and 2 using the algorithmic procedure described in this paper.

\begin{tabular}{|c|c|c|c|c|}
\hline \multirow[t]{3}{*}{$\begin{array}{l}\text { Iteration } \\
\text { No. }\end{array}$} & \multicolumn{4}{|c|}{$\begin{array}{l}\text { Primary structure frequency: } 89.9 \mathrm{~Hz} \\
\text { Primary structure damping ratio: } 0.004\end{array}$} \\
\hline & \multicolumn{2}{|c|}{$\begin{array}{r}\text { Absorber set } 1(n=3) \\
\text { Mass ratio: } 0.11\end{array}$} & \multicolumn{2}{|c|}{$\begin{array}{r}\text { Absorber set } 2(n=1) \\
\text { Mass ratio: } 0.03\end{array}$} \\
\hline & $\begin{array}{l}\text { Tuning } \\
\text { ratio } \alpha\end{array}$ & $\begin{array}{r}\text { Absorber } \\
\text { damping } \\
\text { ratio } \zeta_{1}\end{array}$ & $\begin{array}{l}\text { Tuning } \\
\text { ratio } \alpha\end{array}$ & $\begin{array}{r}\text { Absorber } \\
\text { damping } \\
\text { ratio } \zeta_{1}\end{array}$ \\
\hline 0 & 1 & - & 1 & \\
\hline 1 & 0.780801 & 0.348206 & 0.975460 & 0.093925 \\
\hline 2 & 0.794814 & 0.240212 & 0.975827 & 0.084364 \\
\hline 3 & 0.794323 & 0.245116 & 0.975822 & 0.084489 \\
\hline 4 & 0.794341 & 0.244939 & 0.975822 & 0.084488 \\
\hline 5 & 0.794341 & 0.244945 & 0.975822 & 0.084488 \\
\hline
\end{tabular}

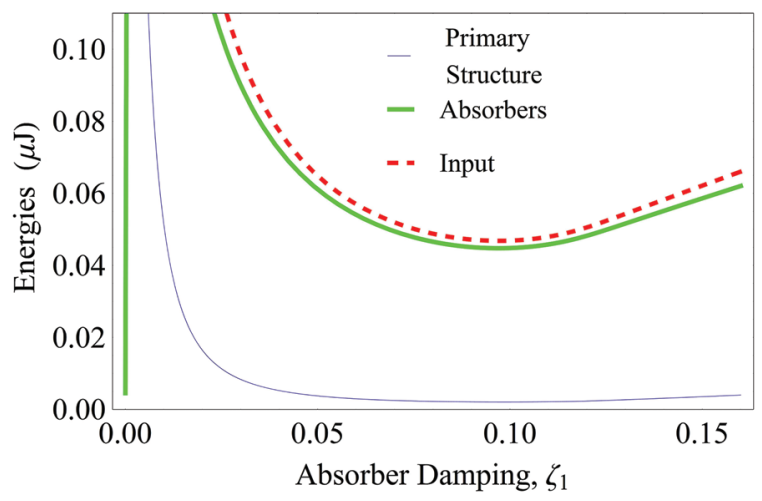

Figure 16: The figure shows the distribution of system energies when frequency ratio is optimized. Much of the input energy is absorbed by the absorbers. Input energy is lowest at the optimal value of absorber damping.

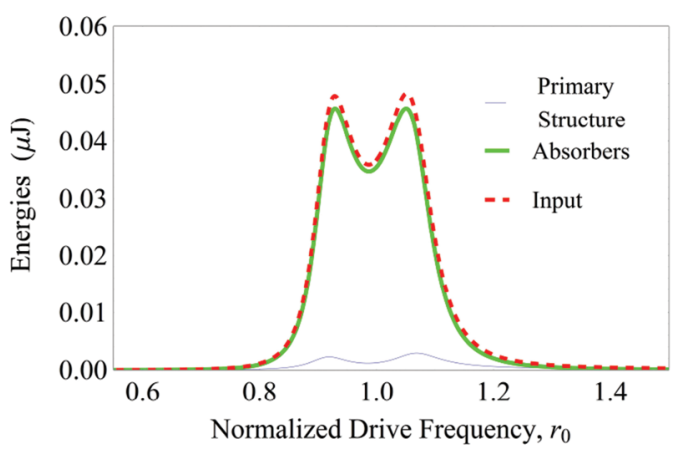

Figure 17: The figure shows the distribution of system energies when both frequency ratio and absorber damping are optimized. This ensures that energy is effectively transferred to harvesters over the designed bandwidth of operation.

The dissipation and input energies in the system operating at the optimal parameters for absorber set 2 with single absorber are shown in Figure 17. It can be noticed that selecting the optimum absorber damping would 
reduce the total energy absorbed by the system. Nevertheless, the correct frequency ratio would ensure that the energy input to the system is taken away by all the absorbers effectively.

\section{Conclusions}

We propose an iterative method to determine the optimum performance parameters for a dynamic vibration absorber system. We derive eigenvalues and eigenvectors, in closed form, for the system consisting of several identical absorbers. Bandwidth of energy absorption is identified as a significant performance parameter to initiate the optimization process. Moreover, it is noticed that bandwidth enforces an upper limit on the mass ratio. Dissipation energies of the absorber array system are derived and used for optimization to determine the optimal values of frequency ratio and absorber damping. The proposed method optimizes the energy absorbed by the system in a very few iterative steps to determine the optimum values. Experiments are performed on cantilever absorbers mounted on a cantilever plate to validate the method. The experimental resonant frequencies and the vibrational response of the system are compared with the anticipated values. The difference in estimating mass ratio and vibrational response is observed to be less than $18 \%$. This is attributed to the approximate functions used to obtain the mass ratio and the vibrational response. Nonetheless, the effects of decreasing the frequency ratio and mass ratio on the bandwidth and the distribution of system energies are verified. Energy absorption is the primary function of energy harvesters. It is essential to design energy harvesters with due consideration to the vibrational characteristics of the primary structure, as this alleviates the system from undesirable energy extraction and enables effective energy absorption over a selected bandwidth. The practical implementation of optimum absorber damping is easier in the context of energy harvesting as the damping in the harvester can be tuned by an external impedance matching circuitry. Hence, the inherent variability associated with damping in energy harvesters can be utilized advantageously to achieve the optimal damping. We emphasize, through this work that monitoring energies in the system and optimizing them is both rational and vital for designing a system of harvesters for a given damped vibrating source. We show that the proposed procedure to obtain the optimum performance parameters is robust and quick for designing multiple absorbers with direct relevance to energy harvesting.
Acknowledgements: The current work is supported by National Program on Micro and Smart Systems grant through the facilities created at Centre for Nano Science and Engineering, Indian Institute of Science. Rammohan expresses earnest gratitude to Mr. Vamsy Godthi for sharing his expertise in experimental setup with vibrometer and several valuable discussions during the course of this research work.

\section{Appendix 1}

The equations of motion for the dynamic vibration absorber system consisting of $n$ identical absorbers and a primary structure are shown in the following equations.

$m_{0} \ddot{x}_{0}+\left(k_{0}+n k_{1}\right) x_{0}-n k_{1} x_{1}+\left(c_{0}+n c_{1}\right) \dot{x}_{0}-n c_{1} \dot{x}_{1}=-m_{0} \ddot{x}_{g}$

$m_{i} \ddot{x}_{i}+k_{i} x_{i}-k_{i} x_{0}+c_{i} \dot{x}_{i}-c_{i} \dot{x}_{0}=-m_{i} \ddot{x}_{g}$

The second equation of eq. [10] consists of $n$ similar equations of motion with $i=1$ to $n$ for $n$ identical absorbers. The response of the primary structure and any of the secondary absorber is given as,

$$
\begin{gathered}
x_{0}=\frac{r_{0}^{2} x_{g}\left(\alpha^{2} \beta-r_{0}^{2}+2 j r_{0} \alpha \beta \zeta_{1}\right)}{\mu+j v} \\
x_{1}=\frac{r_{0}^{2} x_{g}\left(\chi-r_{0}^{2}+2 j r_{0}\left(\zeta_{0}+\alpha \beta \zeta_{1}\right)\right)}{\mu+j v} \\
\text { where } \mu=\left(1-r_{0}^{2}\right)\left(\alpha^{2}-r_{0}^{2}\right)-r_{0}^{2}\left(\alpha^{2} n m_{r}+4 \alpha \zeta_{0} \zeta_{1}\right) \\
\text { and } v=2 r_{0}\left(\zeta_{0}\left(\alpha^{2}-r_{0}^{2}\right)+\alpha \zeta_{1}\left(1-\beta r_{0}^{2}\right)\right)
\end{gathered}
$$

The response of the system as given by eqs [11] and [12] is used in the expressions for system energies described in eqs [3], [4] and [5] to arrive at the conditions for determining optimal values of frequency ratio and absorber damping.

\section{References}

Arrieta, A. F., T. Delpero, A. E. Bergamini, and P. Ermanni. 2013. "Broadband Vibration Energy Harvesting Based on Cantilevered Piezoelectric Bi-Stable Composites.” Applied Physics Letters 102:173904.

Bapat, V. A. and H. V. Kumaraswamy. 1979. "Effect of Primary System Damping on the Optimum Design of an Untuned Viscous Dynamic Vibration Absorber." Journal of Sound and Vibration 63 (4):469-74.

Braun, S. 2002. "DVAs for Viscously Damped Primary Systems" in the Encyclopedia of Vibration, Academic Press, Volume 1, 9-18. (Editors: D. Ewins, and S. S. Rao). 
Brennan, Michael J., Gilberto Pechoto Melo, and Vicente Lopes Jr. 2013. On the simultaneous use of a resonator as an energy harvester and a vibration absorber, 11th international conference on recent advances in structural dynamics, Pisa, Italy, July 1-3.

Chakraverty, S. 2009. "Boundary Characteristic Orthogonal Polynomials (BCOPs) in Vibration of Plates." In The Book Vibration of Plates, 167-91. CRC Press - Taylor and Francis Group, Boca Raton, FL 33487-2742.

Den Hartog, J. P. 1985. "Two degrees of freedom" in the book Mechanical vibrations. New York: Dover Publications, Inc. 93-104.

Esmailzadeh, E. and N. Jalili. 1998. "Optimum Design of Vibration Absorbers for Structurally Damped Timoshenko Beams." Journal of Vibration and Acoustics (ASME) 120:833-41.

Faruque Ali, S. and Sondipon Adhikari. 2013. "Energy Harvesting Dynamic Vibration Absorbers." Journal of Applied Mechanics 80(4):041004 1-9.

Ghosh, A. and B. Basu. June 2007. "A Closed-Form Optimal Tuning Criterion for TMD in Damped Structures." Structure Control Health Monitoring 14(4):681-92

Lee, Jae-Hyeong, Jai-Hyuk Hwang, Jae-Sung Bae, Keunsoo Park, and Daniel J. Inman. 2014. Piezoelectric energy harvester with wide bandwidth using TMD concept, 17th U.S. national congress on theoretical and applied mechanics, Michigan, USA, June 15-20.

Liu, Kefu and Gianmarc Coppola. 2010. Optimal design of damped dynamic vibration absorber for damped primary systems, CSME-38, E.I.C. Accession 3124, 09.
Liu, Kefu and Jie Liu. 2005. "The Damped Dynamic Vibration Absorbers: Revisited and New Result." Journal of Sound and Vibration 284:1181-9.

Najafi, M., M. R. Ashory, and E. Jamshidi. 2009. Optimum configuration for vibration absorbers of a SDOF system using generic algorithm, Society for experimental mechanics Inc. Proceedings of IMAC-XXVII, Orlando, Florida USA, Feb 9-12.

Randall, S. E., D. M. Halsted and D. L. Taylor. 1981. "Optimum Vibration Absorbers for Linear Damped Systems." Journal of Mechanical Design (ASME) 103:908-13.

Thompson, A. G. 1981. "Optimum Tuning and Damping of a Dynamic Vibration Absorber Applied to a Force Excited and Damped Primary System." Journal of Sound and Vibration 77(3):403-15.

Thompson, D. J. 2007. The theory of a continuous damped vibration absorber to reduce broad-band wave propagation in beams, Institute of sound and vibration research, ISVR Technical Memorandum No. 968.

Warburton, G. B. 1982. "Optimal Absorber Parameters for Various Combinations of Response and Excitation Parameters." Earthquake Engineering and Structural Dynamics 10:381-401.

Wong, W. O. and Y. L. Cheung. 2008. "Optimal Design of a Damped Dynamic Vibration Absorber for Vibration Control of Structure Excited by Ground Motion." Engineering Structures 30:282-6. Yang, Jin, Xihai Yue, Yumei Wen, Ping Li, Qiangmo Yu, and Xiaoling Bai. 2014. "Design and Analysis of a 2d Broadband Vibration Energy Harvester for Wireless Sensors." Sensors and Actuators A: Physical 205:47-52. 